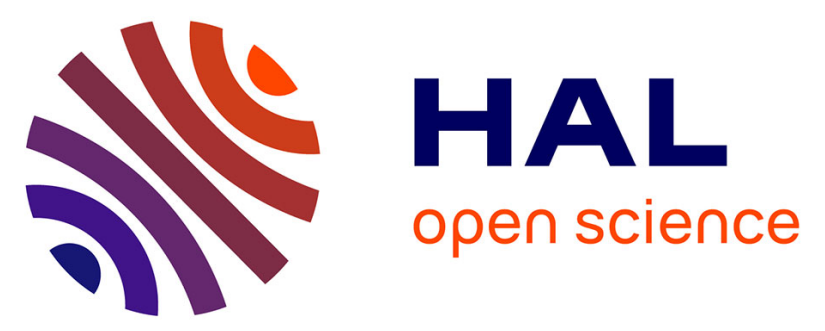

\title{
Digital holographic measurement of the Lagrangian evaporation rate of droplets dispersing in a homogeneous isotropic turbulence
}

Jean-Louis Marié, Thibaut Tronchin, Nathalie Grosjean, Loïc Méès, Onur Can Oztürk, Corinne Fournier, Bernard Barbier, Michel Lance

\section{To cite this version:}

Jean-Louis Marié, Thibaut Tronchin, Nathalie Grosjean, Loïc Méès, Onur Can Oztürk, et al.. Digital holographic measurement of the Lagrangian evaporation rate of droplets dispersing in a homogeneous isotropic turbulence. Experiments in Fluids, 2017, 58, pp.11. 10.1007/s00348-016-2292-8 . hal01447134

\author{
HAL Id: hal-01447134 \\ https://hal.science/hal-01447134
}

Submitted on 26 Jan 2017

HAL is a multi-disciplinary open access archive for the deposit and dissemination of scientific research documents, whether they are published or not. The documents may come from teaching and research institutions in France or abroad, or from public or private research centers.
L'archive ouverte pluridisciplinaire HAL, est destinée au dépôt et à la diffusion de documents scientifiques de niveau recherche, publiés ou non, émanant des établissements d'enseignement et de recherche français ou étrangers, des laboratoires publics ou privés. 


\title{
Digital Holographic measurement of the Lagrangian evaporation rate of droplets dispersing in a homogeneous isotropic turbulence
}

\author{
J.L. Marié - T. Tronchin • N. Grosjean • \\ L. Méès · O. Can Öztürk • C. Fournier • \\ B. Barbier . M. Lance
}

January 9, 2017

\begin{abstract}
The evaporation rate of diethyl ether droplets dispersing in a homogeneous, nearly isotropic turbulence is measured by following droplets along their trajectory. Measurements are performed at ambient temperature and pressure by using in-line digital holography. The holograms of droplets are recorded with a single high-speed camera $(3 \mathrm{kHz})$ and droplets trajectories are reconstructed with an "Inverse problem approach" algorithm (IPA) previously used in Chareyron et al (2012); Marié et al (2014). The thermal-vapor concentration wakes developing around the droplets are visible behind each hologram. A standard reconstruction process is applied, showing that these wakes are aligned with the relative Lagrangian velocity seen by droplets at each instant. This relative velocity is that obtained from the dynamic equation of droplets motion and the positions and diameter of the droplets measured by holography and the IPA reconstruction. Sequences of time evolution of droplets $3 \mathrm{D}$ positions, diameter, and $3 \mathrm{D}$ relative velocity are presented. In a number of cases, the evaporation rate of droplets changes along the trajectory and deviates from the value estimated with a standard film model of evaporation. This shows that turbulence may significantly influence the phase change process.
\end{abstract}

Keywords Lagrangian tracking · evaporation · digital holography · "Inverse problem approach"

\section{Introduction}

In many practical situations droplets vaporize in the turbulent flow carrying them. It is therefore of primary importance to know whether or not this turbulence influences their evaporation (Birouk and Gökalp, 2006; Reveillon and Demoulin, 2007).

J.L. Marié · T. Tronchin · N. Grosjean · L. Méès · O. Can Öztürk · B. Barbier · M. Lance Laboratoire de Mécanique des Fluides et d'Acoustique UMR CNRS 5509, Ecole Centrale de Lyon - Université Claude Bernard Lyon 1 - INSA Lyon,

36 avenue Guy de Collongue, 69134 Ecully cedex, France E-mail: jean-louis.marie@ec-lyon.fr

C. Fournier

Laboratoire Hubert Curien UMR CNRS 5516- Université Jean Monnet 18 rue Pr Benoît Lauras, F-42000, St Etienne, France E-mail: corinne.fournier@univ-st-etienne.fr 
One of the most physically relevant approaches to investigate this question is to adopt a Lagrangian point of view like in turbulent dispersion studies (Pope, 2000; Toschi and Bodenschatz, 2009). It consists in tracking single droplets and measuring the Lagrangian time evolution of their diameter along their trajectories. This is the objective that we aim to achieve. In the experiment currently under progress the droplets are released in a homogeneous isotropic strong turbulence produced by synthetic jets (Goepfert et al, 2010). One advantage of this configuration is to allow a better control of the turbulence parameters. The tracking method which has been chosen is the in-line digital holography because it provides information on size and space position using a rather simple optical device (Gopalan et al, 2008; Lu et al, 2008; Katz and Sheng, 2010; Nguyen et al, 2011). To obtain the accuracy on position and diameters required for the study of evaporation, the holograms recorded are reconstructed with an "Inverse problem approach" (IPA) algorithm (Soulez et al, 2007a,b; Gire et al, 2008; Fournier et al, 2011) that differs from the standard back propagation reconstruction methods. A first experiment with freon droplets (Chareyron et al, 2012) has clearly shown the ability of the in-line digital holography and IPA to accurately track evaporating droplets in the turbulence. However injecting freon proved to be arduous and did not permit to record enough trajectories to detect a potential influence of turbulence on evaporation. This led us to replace freon by diethyl ether, which is easier to inject and evaporates very fast in ambient conditions.

This paper provides the first results obtained in turbulence with this fluid. Typical evaporation rate measured along trajectories are presented. While without turbulence this evaporation rate is close to that predicted by the standard " $d^{2}$ " law (Marié et al, 2014), it may significantly fluctuate and deviate from this law in presence of turbulence. The thermal-vapor concentration boundary layers and wakes visible around each hologram of droplets have also been reconstructed with a standard back propagation method, providing a convincing picture of the vapor film around the droplet. The vapor film is found to follow the orientation of the Lagrangian relative velocity seen at each instant by the droplet along its trajectory. This has been proved by calculating this velocity from the positions and diameter of the droplets measured by holography and the droplets equation of motion, as in Chareyron et al (2012). The results suggest that the fluctuations of the Lagrangian evaporation rate are associated with sudden changes in the Lagrangian relative velocity.

The next section describes the main experimental features: turbulence flow, injection system and optical set-up. The hologram processing (IPA and vapor film standard reconstruction) is presented in Sec. 3. Results are reported and discussed in Sec. 4. The Lagrangian fluid motion around the droplet inferred from their location and diameter is first compared with the reconstructed thermal/vapor wake image. Then a selection of typical trajectories and Lagrangian evaporation rate is shown and the possible origin of the evaporation rate fluctuations examined. The paper ends with a conclusion on the main findings. 
Table 1 Turbulence characteristics.

\begin{tabular}{|c|c|c|c|c|c|c|c|}
\hline \multirow{2}{*}{$\begin{array}{l}\text { twice the } \\
\text { kinetic energy }\end{array}$} & \multirow{2}{*}{$\begin{array}{l}\text { dissipation } \\
\text { rate }\end{array}$} & \multirow{2}{*}{$\begin{array}{l}\text { Taylor } \\
\text { microscale }\end{array}$} & \multirow{2}{*}{$\begin{array}{l}\text { Reynolds } \\
\text { number }\end{array}$} & \multirow{2}{*}{$\begin{array}{l}\text { turnover } \\
\text { time }\end{array}$} & \multirow{2}{*}{$\begin{array}{l}\text { integral } \\
\text { length scale }\end{array}$} & \multicolumn{2}{|c|}{ Kolmogorov } \\
\hline & & & & & & length scale & time scale \\
\hline$q^{2}\left(m^{2} s^{-2}\right)$ & $\epsilon\left(m^{2} s^{-3}\right)$ & $\lambda(\mathrm{mm})$ & $R e_{\lambda}$ & $T_{E}(m s)$ & $\Lambda(m m)$ & $\eta_{k}(m m)$ & $\tau_{k}(m s)$ \\
\hline 2.10 & 7.6 & 5.1 & 229 & 46 & 49 & 0.15 & 1.56 \\
\hline $\begin{array}{l}\text { error estimates } \\
4-5 \%\end{array}$ & $20 \%$ & $11 \%$ & $13 \%$ & $20 \%$ & $15-20 \%$ & $5 \%$ & $9 \%$ \\
\hline
\end{tabular}

\section{Experimental features}

\subsection{Turbulence}

The turbulence is generated by the meeting of three pairs of opposed synthetic jets issued from woofers, as described in Goepfert et al (2010). This system generates a homogeneous, nearly isotropic turbulence whose properties are close to those of the 'box of turbulence' build by Hwang and Eaton (2004). A similar system was recently used in a four pairs configuration by Lian et al (2013). Working in open-chamber conditions ensures that the droplets leaving the turbulence domain without being fully evaporated are hardly re-injected in the turbulence. Moreover, the absence of glass windows is a good choice to avoid any resulting hologram disturbances. To get a fairly good zero mean velocity within the domain, each pair of opposed jets needs to be balanced at the center of the chamber. This is realized by finely tuning the amplitudes of the woofers driving signals using an iterative procedure. As also mentioned in Charalampous and Hardalupas (2010), tiny deviations in the relative amplitude of the driving signals of pairs of opposed jets may cause an imbalance of the flow at the center by displacing the stagnation point.

To control this, integrated silicon differential pressure sensors (Freescale semiconductor MPXV7002 Series) have been inserted in a hole on the outskirts of the perforated plate covering each woofer (Fig. 1a). These transducers convert the pressure oscillations caused by membranes displacement under the perforated plate in a proportional oscillating voltage signal (Fig. 1b). The amplitude of this voltage signal varies linearly with the axial velocity produced by the woofer at the center of the working domain. It is used as feedback in an active control loop to monitor the velocity balance of each pair of jets at the center of the domain. Practically this is done in a Labview environment, by acquiring the pressure signal amplitude of the six woofers in real time on an analogical input module (NI-9234) and continuously correcting the woofer driving signals generated by the analogical output module (NI-9263) . An example illustrating this active control process is given in (Fig. 1c). The pressure signal of each woofer is kept constant within $\pm 0.2 \%$ standard deviation, and hence the axial velocity.

The present runs with ether droplets have been performed in a turbulence having almost the same characteristics as that in Chareyron et al (2012). These characteristics have been measured following the same procedure and are reported in Table 1. The mean flow velocity, the turbulence fluctuations and the integral length scale are of the order of $0.03 \mathrm{~ms}^{-1}, 0.8 \mathrm{~ms}^{-1}$ and $49 \mathrm{~mm}$ respectively. Spatial positioning of the woofers regarding the optical set-up axes is shown in Fig. $2 \mathrm{a}$, b. 


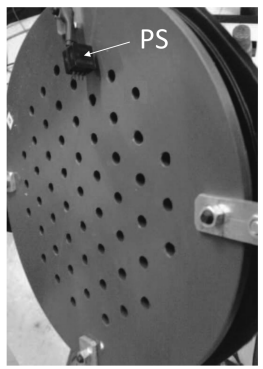

(a)

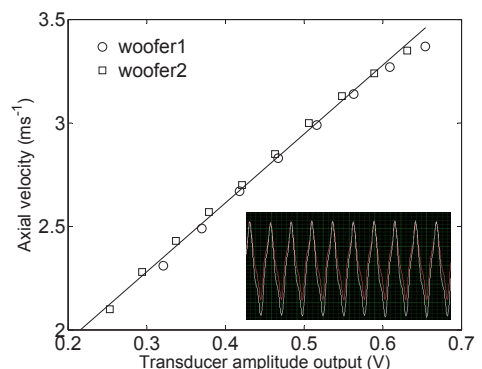

(b)

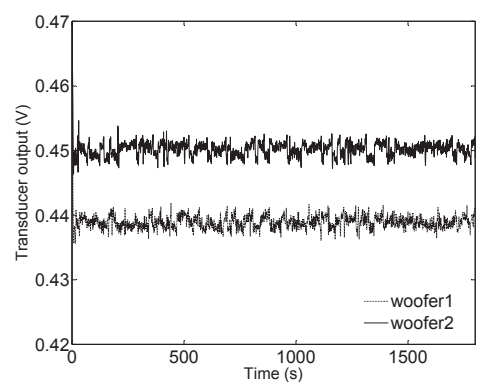

(c)

Fig. 1 a Perforated plate equipped with a pressure sensor (PS). b Calibration curve: axial velocity vs amplitude of the periodic output of the pressure sensor for a pair of facing woofers. Velocity is measured by LDA at half the distance between the woofers. c An example over 30 min of the signal output of the transducers working in active control mode.

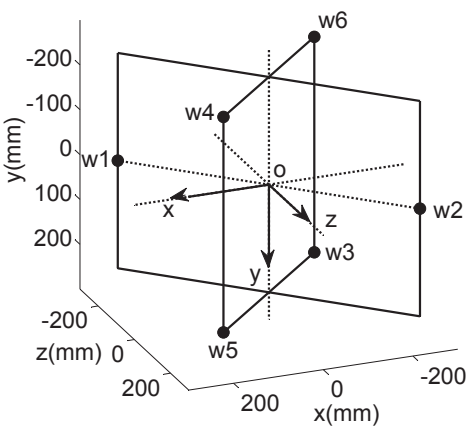

(a)

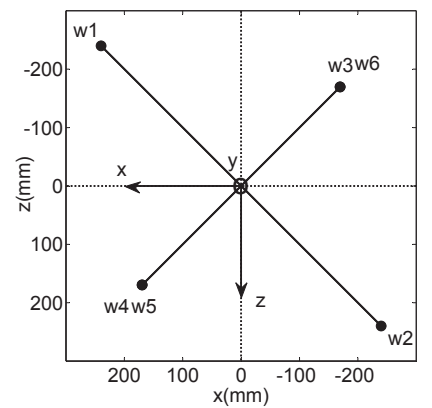

(b)

Fig. 2 a 3D positioning of the woofers and optical set-up axes; distance between woofers of each pair: $680 \mathrm{~mm}$; the angle between each pair is $90^{\circ}$; $\mathrm{z}$ is the optical axis of the in-line holographic set-up, $\mathrm{y}-\mathrm{z}$ are the 2 axes of the 2 components LDA system. b Horizontal plane view.

\subsection{Injection}

The droplets are generated by a piezoelectric MJ-AT-01 MicroFab Technologies jetting device, equipped with a $60 \mu \mathrm{m}$ orifice diameter glass capillary. Unlike in Chareyron et al (2012); Marié et al (2014) where the "drop on demand mode" was used, it is operated in continuous droplet jet-mode. The ether jet created at the capillary exit by a syringe pump is disrupted, which delivers droplets of approximately 1.8 times the orifice diameter (Lee, 2003), that is about $110 \mu \mathrm{m}$, with a velocity of the order of $2 \mathrm{~m} . \mathrm{s}^{-1}$. An example of pdf's of size and velocity measured at the capillary exit by Phase Doppler Anemometry (PDA system: BSA-P60 from Dantec) is displayed in Fig. 3a, b. They are based on a sample of 400,000 droplets fulfilling the sphericity criteria, sufficient to expect an uncertainty for the droplet size distribution of about $3 \%$. Droplets are injected above the turbulence domain at the point $(0,-350 \mathrm{~mm}, 0)$ of the vertical $y$-axis (Fig. 2a, b and Fig. 4). Its distance from the center of the domain was adjusted so that droplets penetrate the turbulence with velocities at maximum equal to the turbulence fluctuations. The 


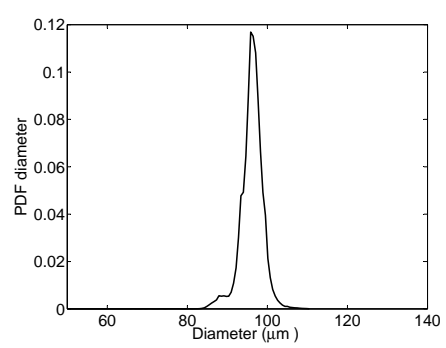

(a)

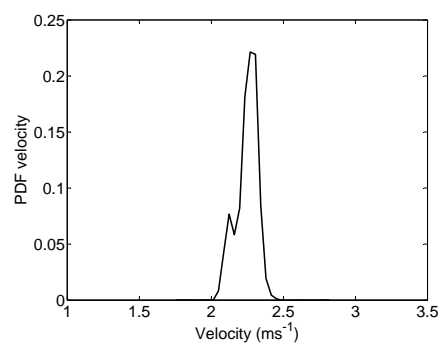

(b)

Fig. 3 Example of pdf's of a: droplet size and b: velocity, as measured with PDA at the capillary exit.

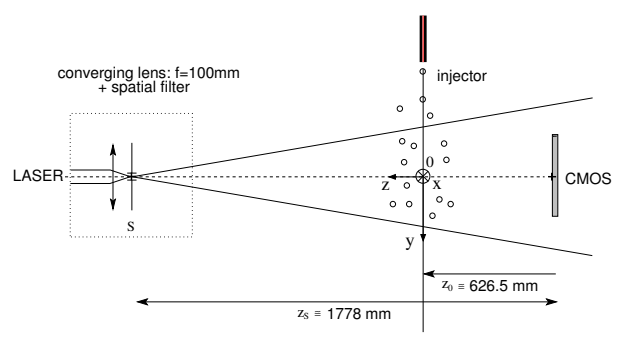

Fig. 4 Optical set-up. The coordinate axis are the same as in Fig. 2

number of droplets recorded on average per hologram within the turbulence never exceeds 10-15, meaning that droplets are sufficiently dilute to consider that they evaporate without interaction. The fluid injected is diethyl ether manufactured by Sigma-Aldrich and containing less than $0.1 \%$ residual water. Droplets are released at ambient temperature and pressure. Air temperature and relative humidity in the room are controlled with an air-conditioner and a dehumidifier, and measured for each run.

\subsection{Optics}

Fig. 4 provides a schematic of the in-line optical set-up. The turbulence domain is illuminated by a laser beam (wavelength $\lambda=532 \mathrm{~nm}$ ) produced by a Nd:YVO4 solid laser Millenia IIs Spectra Physics. To improve the uniformity of the lighting, the beam at the laser exit is focused by a converging lens onto a pinhole placed at 


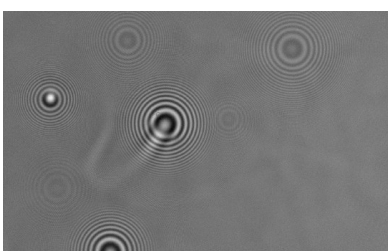

(a)

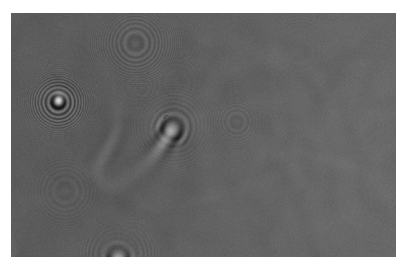

(b)

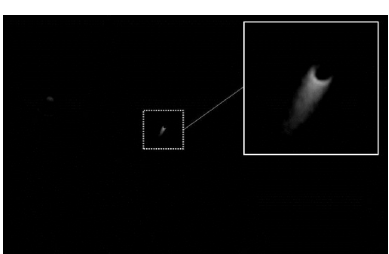

(c)

Fig. 5 a Example of droplets hologram. b Same hologram after subtraction of the best fit model hologram (cleaned hologram). c Cleaned hologram after wake reconstruction.

its focal lens $f=100 \mathrm{~mm}$ and then diverges towards the domain of measurement. The holograms of droplets are recorded at a frequency of $3 \mathrm{kHz}$ on the $800 \times 1280$ pixels CMOS sensor of a Phantom V611 camera, pixel size $20 \mu \mathrm{m}$, fill-factor 0.56 . The sensor is positioned at a distance $z_{0}=626.5 \mathrm{~mm}$ from the center of the turbulence domain $(x=y=z=0$, Fig. 2). Given this configuration, the part of the turbulence domain imaged is a parallelepiped of about $10 \times 16 \times 50 \mathrm{~mm}^{3}$, which is approximately the volume investigated in Chareyron et al (2012). As shown in this reference on water droplets, the use of an "Inverse problem approach" to process the holograms enables to detect droplets outside of the field of view up to an effective cross size as large as $42 \mathrm{~mm} \times 40 \mathrm{~mm}$, of the order of the area of the turbulence domain. This potentiality have not been used here for two reasons: first it is much more time consuming than infield measurements (up to 8-10 times longer in some cases) and more importantly, the thermal/vapour films created by evaporation around the droplets generate holograms modifications (see section 3.2), which renders the outfield detection more delicate and less accurate. The divergence of the beam leads to a magnification ratio $\mathrm{m}$ of the holograms which depends on the $z$ coordinate of the droplet according to the relationship:

$$
m(z)=\frac{z_{s}}{\left(z_{s}-\left(z_{0}+z\right)\right)}
$$

where $z_{s}$ is the distance between the CMOS sensor and the light source $\mathrm{S}$, and $z_{0}$ the distance between the CMOS sensor and the center 0 of the measurement volume. In the present configuration, the magnification ratio is about 1.544 for $z=0$ and it varies between 1.51 and 1.58 in the measurement volume. The magnification factor has been deduced from calibration by using a linear scale glass reticle (Edmunds Optics, $\sharp 62-252$ ), following the same procedure as in Marié et al (2014). Practically, a spherical droplet with coordinates $x, y, z$ and diameter $d$ is therefore reconstructed as an object located at $x_{e}=m(z) x, y_{e}=m(z) y, z_{e}=m(z) z$ with a diameter $d_{e}=m(z) d$.

\section{Hologram processing}

\subsection{D reconstruction}

An "Inverse problem approach" was preferred to a standard back light propagation procedure to extract the coordinates and the diameter of the droplets. The reason is that it provides the high accuracy on droplet position and size that is required 
to track them and to measure their evaporation rate at large distance. The IPA method is described in Soulez et al $(2007 \mathrm{a}, \mathrm{b})$ and has been successfully applied in Chareyron et al (2012); Seifi et al (2013); Marié et al (2014). It consists in minimizing the difference between each recorded hologram and a model hologram. The model that describes a spherical droplet hologram at large distance with enough accuracy is the scalar diffraction model of an opaque disk with Fraunhofer approximation. It only depends on the diameter $d_{k}$ and location $x_{k}, y_{k}, z_{k}$ of the droplets (Born and Wolf, 1980). Minimizing the difference is thus equivalent to find iteratively within a $4 \mathrm{D}$ search space, the set of parameters $x_{k}, y_{k}, z_{k}, d_{k}$ producing the model hologram that best matches each individual droplet hologram on the image. In contrast to the classical back light propagation reconstruction process, the model hologram intrinsically takes into account truncation and low spatial resolution of the sensor (Gire et al, 2008) which are significant sources of error and low accuracy measurements. The accuracy of IPA for tracking droplets in the turbulence under the same operating conditions as the ones here, has been discussed in detail in Chareyron et al (2012). For $60 \mu \mathrm{m}$ diameter water droplets, it has been typically estimated to $1 \mu \mathrm{m}$ on diameter, $3 \mu \mathrm{m}$ (about one sixth pixel size) on the transverse positions (parallel to the sensor) and about $60 \mu \mathrm{m}$ (one diameter) on the depth. The concentration of droplets being low, their tracking from image to image has been achieved by using a simple 3D nearest-neighbor criteria.

\subsection{Thermal/vapor film reconstruction}

Fig. 5a shows an example of droplet hologram recorded in the in-line configuration depicted in Fig. 4. Droplets holograms consist of circular fringes resulting from interferences between the droplet diffraction pattern and the reference beam which is the illuminating beam it-self. A wake, attached to the central hologram is also clearly visible. It is caused by the deflection of the light by the refractive index gradient in the thermal - vapor concentration boundary layers and wakes developing around the droplet as discussed in Seifi et al (2013); Méès et al (2013). It was shown that the wake image visualizes well the Lagrangian relative motion of the air around the droplet (Chareyron et al, 2012; Marié et al, 2014). The effect of the vapor is more pronounced in the center part of the hologram and is not taken into account in the hologram Fraunhofer formation model. This is why a weighted mask is generally used to exclude the central part of the hologram for the $3 \mathrm{D}$ reconstruction process (Chareyron et al, 2012; Seifi et al, 2013; Marié et al, 2014). To get more information on the thermal/vapor wake and its evolution in time (when considering a sequence of holograms), the best fit model hologram obtained by IPA can be subtracted from the initial image as shown on the example in Fig. 5b. We assume that the resulting gray scale variation around the droplet thus obtained is only due to the thermal/vapor wake generated by the droplet evaporation. It is then considered as a phase object hologram and it is reconstructed using a standard back light propagation method to obtain the image shown in Fig. 5c. The distance $z$ used for this reconstruction is that obtained from IPA measurement, i-e in the plane of the droplet. Under that form the edges of the wake in the sensor plane are reconstructed at the droplet scale and so, can be more easily compared with the projection in the plane of the Lagrangian relative velocity (see section 
4.1). Note that a threshold has been applied to the image in Fig. 5c to remove the twin image noise which is a well known drawback of the standard back light propagation method used in this last part of the image processing.

\section{Results}

4.1 Lagrangian fluid motion around the droplets

As shown in Chareyron et al (2012), it is possible to calculate theoretically the Lagrangian relative velocity seen at each instant by the droplet from its position and diameter measured by digital holography. It requires that the main forces acting on the droplets reduce to the gravity and the drag force, in which case their motion is described by an equation of the form (see for instance Michaelides (2006))

$$
\frac{d \mathbf{u}_{d}}{d t}=\left(1-\frac{1}{\gamma}\right) \mathbf{g}+\frac{3 C_{D}\left(R e_{d}\right)}{4 d \gamma}\left|\mathbf{u}-\mathbf{u}_{d}\right|\left(\mathbf{u}-\mathbf{u}_{d}\right)
$$

where $\gamma$ is the ratio of densities $\rho_{d} / \rho_{g}, \mathbf{u}_{d}$ the droplet velocity, $\mathbf{u}$ the gas velocity and $R e_{d}$ the Reynolds number based on the droplet diameter and the relative velocity $\mathbf{u}_{r}=\mathbf{u}-\mathbf{u}_{d}$. Here, this form is justified because the droplets are heavier than the gas, have a size comparable to the Kolmogorov length scale $\eta_{k}$ and do not interact during evaporation, since their concentration is low. The diethyl ether droplets having a Reynolds number typically in the range $0 \leq R e_{d} \leq 40$, the drag coefficient is estimated by the correlation proposed by Clift et al (1978) for this case

$$
C_{D}\left(R e_{d}\right)=\frac{24}{R e_{d}}\left(1+\beta R e_{d}^{\alpha}\right)
$$

where $\beta=0.1935$ and $\alpha=0.6305$. The unknown Lagrangian relative velocity norm $u_{r}$ is then inferred by solving the equation obtained by summing the square of the projections of equation (2) along the three directions of the holographic set-up:

$$
\tau_{d}^{2}\left(\dot{u}_{d x}^{2}+\left(u_{d y}-g\right)^{2}+u_{d z}^{2}\right)=u_{r}^{2}\left(1+\beta R e_{d}^{\alpha}\right)^{2}
$$

where $\tau_{d}=\rho_{l} d^{2} / 18 \mu_{g}$ is the droplet time response and symbol (') denotes the time derivative.

Finding $u_{r}$, solution of Eq. (4), requires two conditions. The drag model must be physically relevant and the velocities, accelerations and diameters must be estimated with a high accuracy. IPA provides this accuracy on diameter and transverse positions, but not directly on the depth positions. The measured $z$ coordinates are therefore filtered (smoothed), as explained in Chareyron et al (2012), to get a comparable accuracy on depth velocities and accelerations. Practically, $\tau_{d}, u_{d x}{ }^{2}$, $u_{d y}{ }^{2}, u_{d z}{ }^{2}$ are calculated at each time step $(0.33 \mathrm{~ms})$ from the reconstructed trajectory and the measured diameter. Once $u_{r}^{2}$ has been determined, $R e_{d}$ is known and the three components of the relative velocity can be readily deduced from the 3 projections of equation (2). For a better result, the estimation of $\mathbf{u}_{r}$ by this method has been implemented in the simple model of evaporation/condensation 

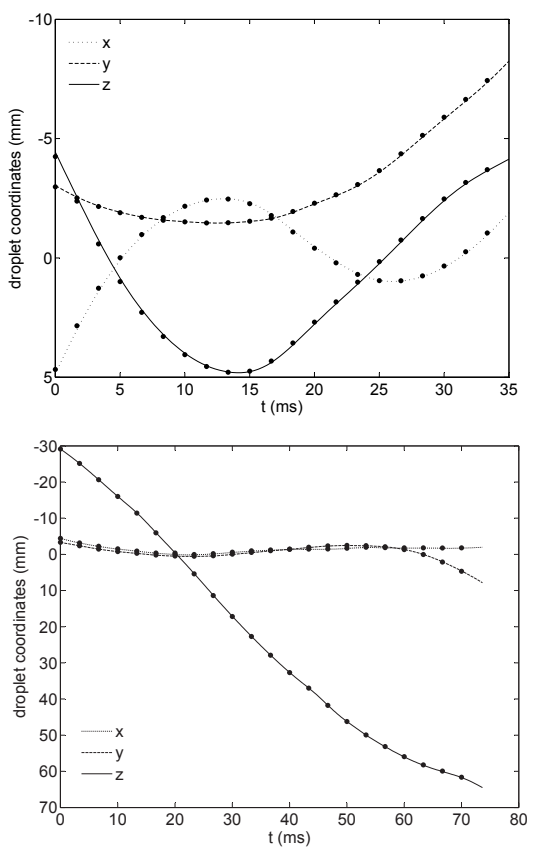

(a)

(b)

Fig. 6 Two examples of trajectory measured by holography (closed circles) and calculated from the relative velocity determined from the measurements (lines). a: traj1, b: traj10 (see Fig. 10a for 3D plot).

proposed in Marié et al (2014) for ether droplets falling in quasi-still air. $u_{r}$ is then determined from the fluid properties given by the model at each iteration and can be used to see how convective effects influence the vaporizing rate and the heat transferred to the droplet (Eq. (7) and (11) in Marié et al (2014)). The Reynolds number $R e_{d}$ used in Eq. (3), (4), is that based on the viscosity $\mu_{g}^{r}$ of the mixture of humid air and ether vapor around the droplet in the reference conditions, and the density of humid air at infinite $\rho_{h a}^{\infty}$ :

$$
R e_{d}=\frac{\rho_{h a}^{\infty}\left(u_{d}-u\right) d}{\mu_{g}^{r}}
$$

The transfers between the droplets and its surrounding are expressed in the frame of the double film theory (Bird et al, 1960), like in Abramzon and Sirignano (1989). All processes are supposed to be quasi-steady, and the thermal and concentration boundary layers developing around the droplets are modeled by two spherical films, whose thicknesses account for the Stefan flux and the convective effects of the external flow. The knowledge of $u_{r}$, hence of $R e_{d}$ from DH measurements enables calculation of the Sherwood $S h_{0}$ and Nusselt $N u_{0}$ numbers describing the mean convective effects within these boundary layers through the correlation of Clift et al (1978):

$$
\begin{aligned}
S h_{0} & =1+\left(1+R e_{d} S_{c g}\right)^{\frac{1}{3}} f\left(R e_{d}\right) \\
N u_{0} & =1+\left(1+R e_{d} P_{r g}\right)^{\frac{1}{3}} f\left(R e_{d}\right)
\end{aligned}
$$


where $f\left(R e_{d}\right)=1$ for $R e_{d} \leq 1$ and $f\left(R e_{d}\right)=R e_{d}^{0.077}$ for $R e_{d} \leq 400$. $P_{r g}$ denotes the Prandt number, $S_{c g}$ the Schmidt number of the gas. It is therefore possible to check whether the implementation of the relative velocity in this quasi-steady approach can reproduce the measured vaporization rates (section 4.2).

Droplets trajectories measured by holography have been systematically compared to those calculated with the model thus modified. Fig. 6a shows the droplet coordinates of one of them (referenced as traj1). Clearly, the measured and calculated droplets coordinates superpose rather well. This evidences that the relative velocity is correctly estimated at each time step from holographic measurements (positions and diameter) and that using this velocity in Eqs $(2-3,5)$, together with the measured diameter, can reproduce the trajectory satisfactorily. The same good agreement is obtained for traj10 with greater displacement in depth-direction $\mathrm{z}$ (Fig. 6b). This shows that the $z$ filtering mentioned above compensates the lower accuracy in this direction efficiently. The $x-y$ projection of this Lagrangian relative velocity, $\mathbf{u}_{r x y}$, is compared in Fig. 7 with the $x-y$ reconstructed thermal/vapor wake image. Angles are referenced according to the sketch in the right corner bottom image. Several points are worth noting. Whatever the image (hologram or reconstruction), the wake is most of the time clearly aligned with $\mathbf{u}_{r x y}$, thus confirming the results obtained with freon droplet (Chareyron et al, 2012). This is still more obvious on the reconstruction view, where the film of vapor is reconstructed at the droplet scale and the axis of the wake can be determined more precisely (white dotted line). The axis is defined as the line joining the center of the droplet and the barycenter of the vapor film. It's length in pixels $L_{w p i x}$ is proportional to the length of the reconstructed wake and its angle $\theta_{w x y}$ gives its orientation. The orientation of this line is seen to follow rapidly the orientation of $\mathbf{u}_{r x y}$ even between $\mathrm{t}=17$ and $20 \mathrm{~ms}$, where $\mathbf{u}_{r x y}$ rotates almost pi radians very quickly. However, the wake does not realign instantaneously with the relative velocity, but with a certain time delay that corresponds to the response time $\tau_{w}$ of the wake to changes in $\mathbf{u}_{r x y}$ direction. This appears in Fig. 8a where the temporal evolution of the angles $\theta_{w x y}$ of the wake axis and $\theta_{u r x y}$ of the $\mathbf{u}_{r x y}$ direction in the $x-y$ plane are plotted. After each new change in $\mathbf{u}_{r x y}$ direction, the relative flow in the plane $x-y$ needs a convective time

$$
\tau_{r x y} \approx \frac{d}{u_{r x y}}
$$

to pass around the droplet, hence it seems reasonable to think that $\tau_{w}$ is related to $\tau_{r x y}$. Supposing that $\theta_{w x y}$ responds linearly to $\theta_{\text {urxy }}$ with a time $\tau_{w}$ proportional to $\tau_{r x y}$, then $\theta_{w x y}$ should vary as

$$
\frac{d \theta_{w x y}}{d t}=\frac{\theta_{u r x y}-\theta_{w x y}}{\tau_{w}} ; \tau_{w}=k \times \tau_{r x y}
$$

where $\mathrm{k}$ is a constant to be estimated and $\tau_{r x y}$ the Lagrangian convective time inferred from $d$ and $u_{r x y}$ at each time step.

A good matching between Eq. (8) and the angle of the reconstructed wake of traj 1 is found when taking $\mathrm{k} \approx 3$. In this example, the average response time $\bar{\tau}_{w}$ is typically of the order of $0.4 \mathrm{~ms}$, that is about 8 times smaller than $\bar{\tau}_{d}$. The alignment of the wake with the relative velocity was observed for all trajectories with $\bar{\tau}_{w}$ ranging from 0.3 to $1 \mathrm{~ms}$, and in all cases the angle of the reconstructed wake proved to be satisfactory reproduced by Eq. (8) with the same constant 

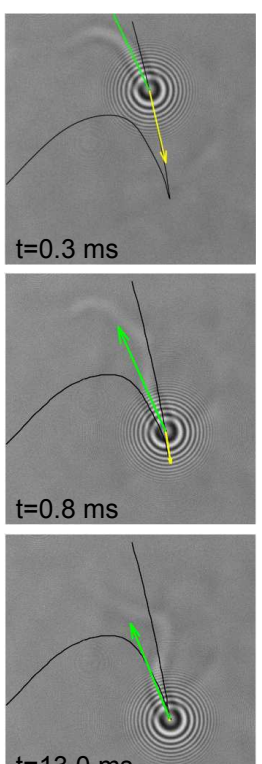

$\mathrm{t}=13.0 \mathrm{~ms}$
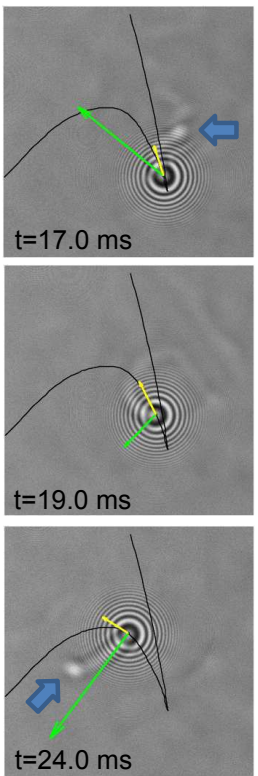
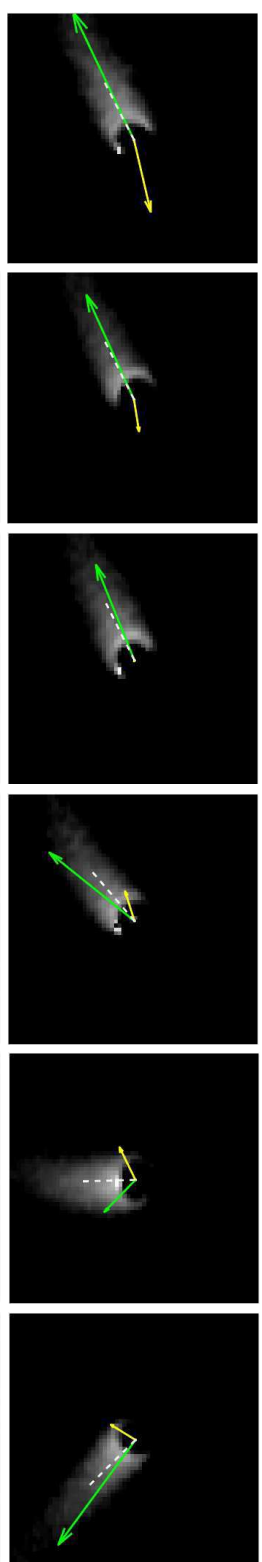
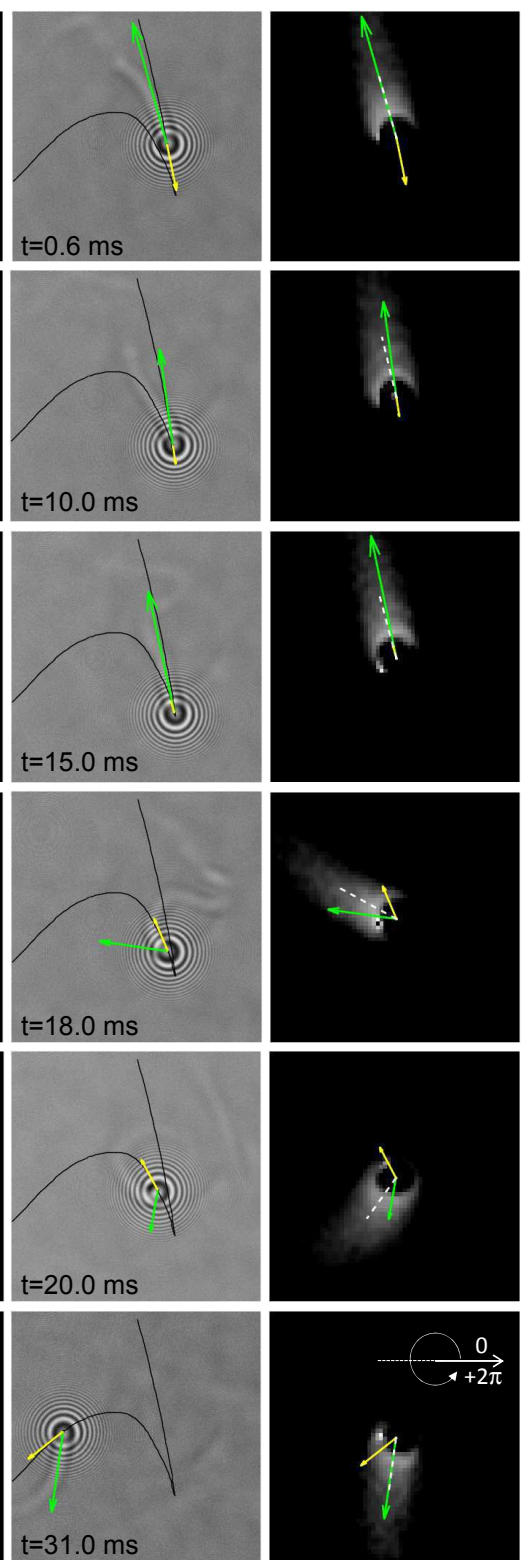

Fig. 7 Time sequence images corresponding to traj1. In grey (first and third column): droplet hologram recorded on the CMOS; the black line is the trajectory of the droplet in the $x-y$ plane; the yellow arrow is the velocity of the droplet $\left(\mathbf{u}_{d x y}\right)$, the green one is the $x-y$ Lagrangian relative velocity $\left(\mathbf{u}_{r x y}\right)$ calculated from the model. In black (second and fourth column): the reconstructed wake, centered on the droplet, with the same two velocities; the white dotted line materializes the axis of the wake; the two blue arrows point out the vapor releases. 

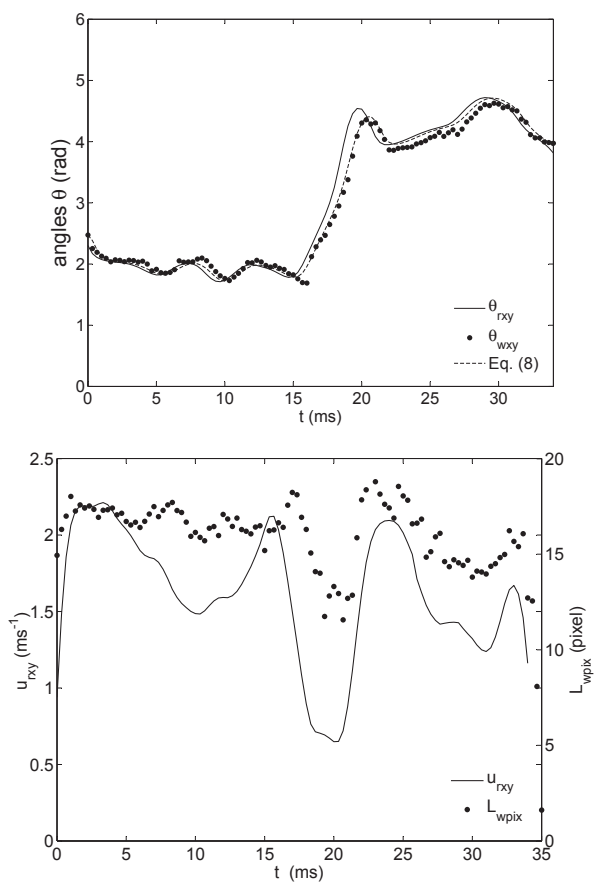

Fig. 8 Comparison of Lagrangian relative velocity $\mathbf{u}_{r x y}$ - 2D reconstructed wake: a time evolution of orientations (see Fig. 7 last image for the sign convention), $\mathbf{b}$ time evolution of $L_{w p i x}$ and $u_{r x y}$.

$\mathrm{k} \approx 3$. Although it is too early to draw any statistical conclusions, these results suggest that $\tau_{w}$ is the necessary delay for the boundary layers around the droplet to reorient after any change of relative flow direction and that it tends to increases as $u_{r x y}$ decreases. Also interesting is to note that the length of the reconstructed wake is qualitatively rather well correlated to $u_{r x y}$ : it increases as $u_{r x y}$ increases. This appears when $L_{w p i x}$ is compared to the variations of $u_{r x y}$ (Fig. 8b). It is remarkable that the two fast increases in $u_{r x y}$ observed at $\mathrm{t}=16$ and $24 \mathrm{~ms}$ are accompanied by a vapor released behind the droplet, visible on holograms at $\mathrm{t}=17$ and $24 \mathrm{~ms}$ (blue arrows in Fig. 7). A 3D representation of traj1 and $\mathbf{u}_{r}$ calculated with the model is shown in Fig. 9. The evolution of $\mathbf{u}_{r}$ thus gives an idea on the way the wake would behave in $3 \mathrm{D}$.

\subsection{Sample of trajectories - Lagrangian evaporation rates}

A sample of trajectories whose characteristics are summarized in Tab. 2 is reported in Fig. 10a. The limits of the turbulence domain in the depth ( $z$ direction) have been materialized by the 2 square faces. As can be seen, all trajectories remain inside the turbulence domain except for the longer one (traj10), which ends outside the domain. They are associated to different behaviors: droplets crossing the domain (traj2, traj4, traj10), droplets changing direction suddenly (traj1, traj6, traj8) or more progressively (traj3, traj5, tra7, traj9). The corresponding time evo- 


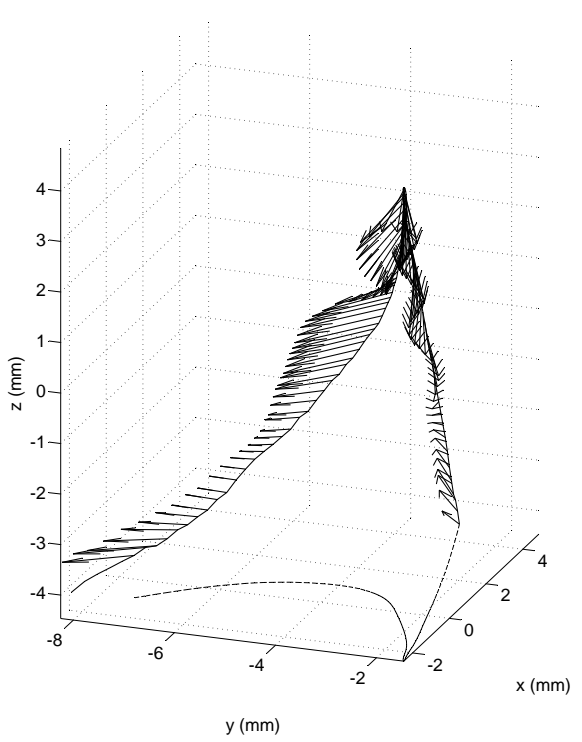

Fig. 93 D plot of traj1 (solid line). The black arrow is the relative Lagrangian velocity calculated with the model. $x-y$ trajectory projection (dashed line).

Table 2 Trajectories - droplets characteristics.

\begin{tabular}{llllll}
\hline $\begin{array}{l}\text { Trajectory } \\
\text { number }\end{array}$ & $\begin{array}{l}\text { Ambient } \\
\text { temperature }\end{array}$ & $\begin{array}{l}\text { Relative } \\
\text { humidity }\end{array}$ & $\begin{array}{l}\text { Initial } \\
\text { diameter }\end{array}$ & $\begin{array}{l}\text { Response } \\
\text { time }\end{array}$ & $\begin{array}{l}\text { Turbulent } \\
\text { Stokes number }\end{array}$ \\
\hline traj $n^{\circ}$ & $T^{\infty}\left({ }^{\circ} \mathrm{C}\right)$ & $R_{h}$ & $d_{0}(\mu \mathrm{m})$ & $\bar{\tau}_{d}(\mathrm{~ms})$ & $\frac{\bar{\tau}_{d}}{\tau_{k}}$ \\
\hline traj1 & 25.5 & 0.33 & 114.5 & 32.1 & $\approx 20$ \\
traj2 & 24.4 & 0.36 & 69.3 & 11.6 & $\approx 7$ \\
traj3 & 24.5 & 0.36 & 82.0 & 15.8 & $\approx 10$ \\
traj4 & 25.2 & 0.34 & 98.5 & 24.3 & $\approx 15$ \\
traj5 & 25.2 & 0.37 & 86.5 & 17.9 & $\approx 11$ \\
traj6 & 23.0 & 0.41 & 80.7 & 15.6 & $\approx 10$ \\
traj7 & 22.9 & 0.41 & 99.0 & 24.3 & $\approx 15$ \\
traj8 & 22.4 & 0.44 & 67.6 & 10.3 & $\approx 6$ \\
traj9 & 27.2 & 0.47 & 106.0 & 28.0 & $\approx 18$ \\
traj10 & 27.2 & 0.47 & 115.0 & 30.0 & $\approx 19$ \\
\hline
\end{tabular}

lution of the square of droplets diameter measured along each trajectory is plotted in Fig. 10b (color points). It is compared with simulation results (continuous color lines) obtained when the relative velocity $u_{r}$ inferred from holographic measurements using Eq. (2) is introduced in Eqs. (6). Simulations have been performed supposing that, at initial time, droplets have reached their equilibrium evaporation temperature for the ambient room conditions. Such an assumption is realistic since droplets are not directly released in the turbulence. Various trends are visible. In some cases (traj2, traj7, traj8, traj10) measurements closely follow the simulation, while in other cases (traj1, traj5) they significantly deviate. The reasons of theses deviations are not yet clearly established. The trends appear more clearly when 
looking at the Lagrangian evaporation rate:

$$
L_{e r}=\frac{-d\left(d^{2}\right)}{d t}
$$

The implementation of $\mathbf{u}_{r}$ calculated from the holographic measurements in the convective terms Eqs. (6) makes that $L_{e r}$ is not constant, as for a standard $d^{2}$ law, but follows the amplitude variations of this relative velocity. Fig. 11a, b show the case of two droplets with about the same initial diameter and close ambient room temperatures. Experimental evaporation rates were calculated by time derivation of the smoothed $d^{2}$ data of Fig. 10b. This smoothing was achieved by cubic splines (function "csap" Matlab) with a smoothing parameter chosen so as to filter the $d^{2}$ variations lower than measurements uncertainty. For traj10 (Fig. 11a), the measured $L_{e r}$ is roughly constant, close to the calculated one. In contrast, it exhibits strong fluctuations for traj1 (Fig. 11b). This is particularly clear for $t$ between 16 and $25 \mathrm{~ms}$, where two sharp increases separated by a sudden decrease takes place at 19 and $23 \mathrm{~ms}$. As mentioned above, the increases coincide with a vapor released behind the droplet (blue arrows Fig. 7). This behavior seems correlated with similar variations of $u_{r}$ over the same period. However, these $u_{r}$ variations are not exactly synchronized in time and the $L_{e r}$ fluctuations they produce in the simulation are very weak compared to the real ones. This shows that this kind of modeling is not adapted to predict this type of event. This is hardly surprising since the modeling is based on quasi-steadiness assumptions and mean values of heat/mass transfer coefficients within boundary layers (Clift et al, 1978). Referring to Fig. 8, these high $L_{e r}$ fluctuations take place in a period where $u_{r x y}$ also strongly rotates, suggesting that the instantaneous $3 \mathrm{D}$ orientation of $\mathbf{u}_{r}$ (see Fig. 9) is also an important parameter. This is confirmed for instance in Fig. 11c where the variations of evaporation rate of traj3 qualitatively follows the variations of the angular velocity of $\mathbf{u}_{r}$ in the plane $x-y$. The flow about droplets being unsteady, it seems reasonable to think that rapid time changes in $\mathbf{u}_{r}$ direction may influence the evaporation rate by suddenly changing the direction of the thermal, vapor, dynamics boundary layers developing around the drop interface (Sirignano, 2010). At some point in Fig. 11b, c the evaporation rate becomes null. Given the accuracy on $d$ expected with IPA: $\delta d= \pm 0.5 \mu \mathrm{m}$ (subsection 3.1), it can hardly be attributed to a measurement uncertainty. Uncertainty on evaporation rate is that on $d^{2}$ difference, that is about $4 d \delta d$, which yields a relative uncertainty on the evaporation rate of about $3 \%$ for a $60 \mu \mathrm{m}$ droplet diameter. The null evaporation rate therefore indicate that evaporation stops or is blocked. Such a behavior have not yet been elucidated. Various effects may be involved. A saturation of vapor concentration around the droplet, as well as the freezing of the humid air condensing at the droplet surface (Marié et al, 2014) could be at the origin of the phenomena. The blocking of evaporation at the surface of droplets is a phenomenon that was already reported by Knubben and van der Geld (2001). It concerned $n$-butane droplets and was attributed in this case to the condensation of humid air and/or the formation of hydrates crystals at the surface.

The number of trajectories presented is obviously too small to allow a statistic analysis of the factors causing the deviation from classic " $d^{2} "$ law. The intense events characterized by strong $\mathbf{u}_{r}$ fluctuations like those along traj1 and traj5 clearly enhance the Lagrangian evaporation rate. They yield evaporation rates which are on average higher than those calculated from the quasi-steady film 


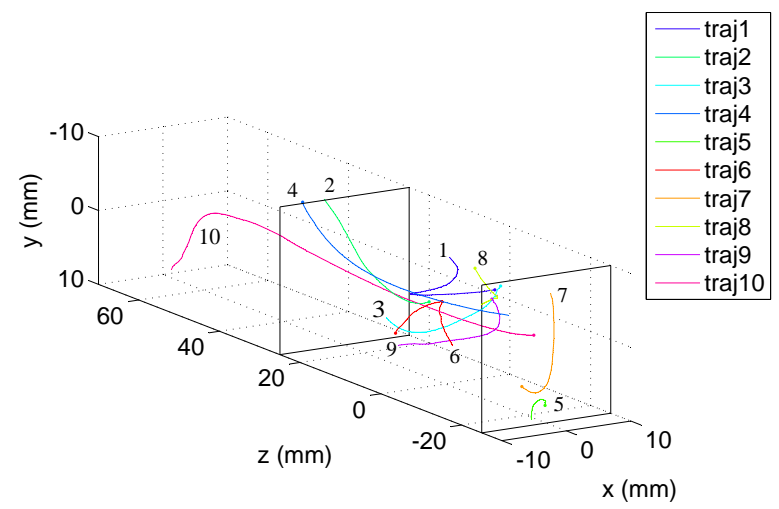

(a)

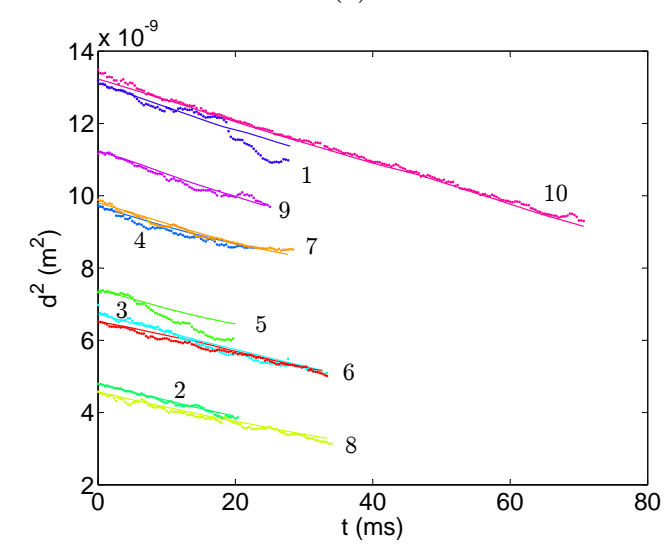

(b)

Fig. 10 a A sample of 3D droplet trajectories; the points are the initial droplet positions, the 2 squares are the $z$ limits of the turbulence domain. $\mathbf{b}$ Time evolution of the diameter square of the droplets along their trajectories; points: DH measurements, continuous color lines: predictions with convective effects modeled by Eq. (6).

modeling and the relative velocity deduced from positions and size of the droplets (Fig. 12). However they are not numerous in the reconstructed trajectories. These events need to be more systematically investigated and understood. This should be the objective of the future work. Likewise, without longer trajectory (tracking time), it is difficult to know how such $\mathbf{u}_{r}$ fluctuations will affect the evaporation law over the droplets lifetime. This requires to enlarge the imaging field of view in the plane $x-y$, which might be realized by modifying the optical set-up.

\section{Conclusion}

Digital in-line holography has been used with a high speed camera to track ether droplets dispersing in a quasi homogeneous and isotropic turbulence and measure their Lagrangian evaporation rate. The droplets holograms recorded on the sensor 

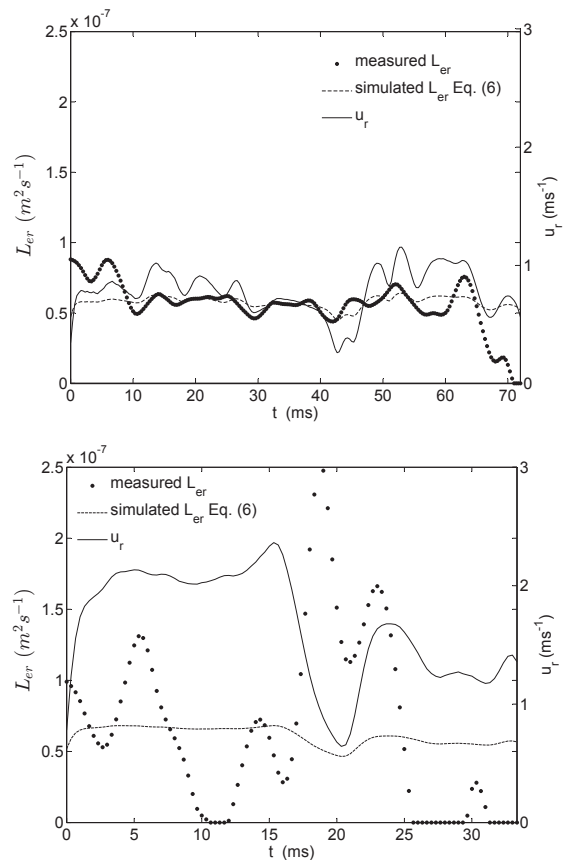

(a)

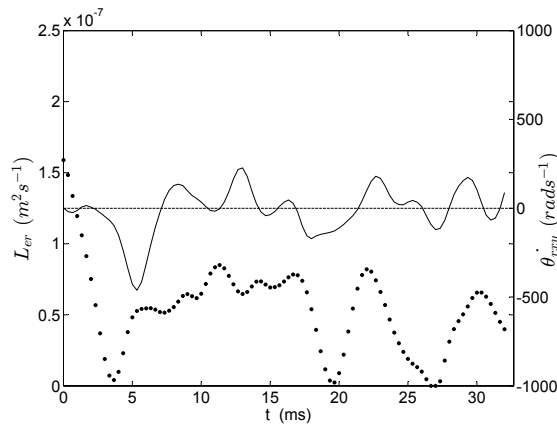

(b)

(c)

Fig. 11 Lagrangian evaporation rates of a: traj10 and b: traj1 compared with the norm $u_{r}$ of the Lagrangian relative velocity. c Evaporation rate of traj3 compared to the angular velocity of $\mathbf{u}_{r}$ in the plane $x-y$

have been processed using an "Inverse problem approach" (IPA), that provides the accuracy on the droplets time positions and diameters required for this study.

The evaporation manifests through the presence of a thermal/vapor wake behind each droplets, thus confirming early observations (Chareyron et al, 2012). This wake can be reconstructed using a standard back light propagation method, once the model that best fits the hologram has been subtracted. The wake thus reconstructed at the scale of the droplet has been compared to the relative velocity deduced from the droplet trajectories and diameters measured by holography via the equation of motion. It is found to follow the instantaneous orientation of relative velocity seen by the droplet along its trajectory, with an average delay that varies between 0.3 and $1 \mathrm{~ms}$, depending on the trajectory. This delay appears as 


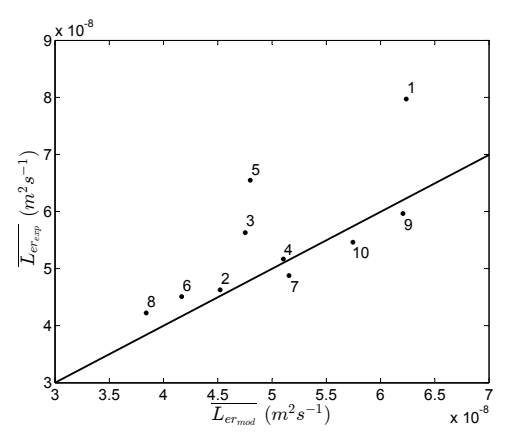

Fig. 12 Experimental vs calculated mean Lagrangian evaporation rates for the ten trajectories. The two highest correspond to traj1 and traj5, respectively.

the time response necessary for the boundary layers around the droplet to adapt to any change of relative flow direction.

The measured Lagrangian evaporation rates are found to behave differently from one trajectory to another. It may be relatively constant as expected for steady equilibrium conditions or strongly vary showing a significant effect of turbulence. In the first case its value is rather well predicted by the simple quasi-steady evaporation/condensation model validated in free falling conditions and the relative velocity calculated from the droplet motion. In the second case, its variations are far stronger than those obtained by simulations, which means that this kind of modeling is not adapted to describe these large fluctuation rates. Data analysis suggests that high fluctuation rates are connected with fast variations of the relative velocity, in magnitude and orientation. However, further investigations and a larger amount of statistics are required to assess this assumption and to clearly identify the involved mechanisms. At this stage, it is difficult to determine whether these strong fluctuations will change the evaporation law over the droplets lifetime.

Acknowledgements This work has been founded by the ANR program TEC2 (Turbulence Evaporation and Condensation). The holographic IPA developments have been performed in the frame of the MORIN project (3D Optical Measurements for Research and INdustry) and supported by the "Programme Avenir Lyon Saint-Etienne" of Lyon University in the framework of "investissement d'avenir" (ANR-11-IDEX-0007).

\section{References}

\section{References}

Abramzon B, Sirignano WA (1989) Droplet vaporization model for spray combustion calculations. Int J Heat Mass Transf 32(9):1605-1618

Bird RB, Stewart WE, Lightfoot EN (1960) Transport Phenomena. John Wiley

Birouk M, Gökalp I (2006) Current status of droplet evaporation in turbulent flows. Prog Energ Combust Sci 32(4):408-423

Born M, Wolf E (1980) Principles of Optics. Pergamon press 
Charalampous G, Hardalupas Y (2010) Clustering of mono-disperse and polydisperse particles in a box of turbulence. In: 7th Int. Conf. Multiphase Flows, Tempa, FL USA

Chareyron D, Marié JL, Fournier C, Gire J, Grosjean N, Denis L, Lance M, Méès L (2012) Testing an in-line digital holography "inverse method" for the Lagrangian tracking of evaporating droplets in homogeneous nearly-isotropic turbulence. New J Phys 14, 043039

Clift R, Grace JR, Weber ME (1978) Bubbles, Drops and Particles. Academic Press

Fournier C, Denis L, Thiébaut E, Fournel T, Seifi M (2011) Inverse problems approaches for digital hologram reconstruction. In: Three Dimensional Imaging, Visualization, and Display 2011, Orlando, United States, vol 8043, pp 1-14

Gire J, Denis L, Fournier C, Thiébaut E, Soulez F, Ducottet C (2008) Digital holography of particles: benefits of the "inverse-problem" approach. M Sci Technol 19(7), 074005

Goepfert C, Marié JL, Chareyron D, Lance M (2010) Characterization of a system generating a homogeneous isotropic turbulence field by free synthetic jets. Exp Fluids 48(5):809-822

Gopalan B, Malkiel E, Katz J (2008) Experimental investigation of turbulent diffusion of slightly buoyant droplets in locally isotropic turbulence. Phys Fluids 20(9), 095102

Hwang W, Eaton JK (2004) Creating homogeneous and isotropic turbulence without a mean flow. Exp Fluids 36:444-454

Katz J, Sheng J (2010) Applications of holography in fluid mechanics and particle dynamics. Annu Rev Fluid Mech 42:531-555

Knubben G, van der Geld CWM (2001) Drop size distribution evolution after continuous or intermittent injection of butane or propane in a confined air flow. Applied Thermal Engineering 21:787-811

Lee ER (2003) Microdrop generation. CRC Press

Lian H, Charalampous G, Hardalupas Y (2013) Preferential concentration of polydispersed droplets in stationary isotropic turbulence. Exp Fluids 54:1525, doi 10.1007/s00348-013-1525-3

Lu J, Fugal JP, Nordsiek H, Saw EW, Shaw RA, Yang W (2008) Lagrangian particle tracking in three dimensions via single-camera in-line digital holography. New J Phys 10, 125013

Marié JL, Grosjean N, Méès L, Seifi M, Fournier C, Barbier B, Lance M (2014) Lagrangian measurements of the fast evaporation of falling diethyl ether droplets using in-line digital holography and a high speed camera. Exp Fluids 55(4):1708, doi:10.1007/s00348-014-1708-6

Méès L, Grosjean N, Chareyron D, Marié JL, Seifi M, Fournier C (2013) Evaporating droplet hologram simulation for digital in-line holography setup with divergent beam. J Opt Soc Am A 30(10):2021-2028

Michaelides EE (2006) Particles, bubbles and drops: Their Motion, Heat \& Mass Transfer. World Scientific

Nguyen D, Honnery D, Soria J (2011) Measuring evaporation of micro-fuel droplets using magnified DIH and DPIV. Exp Fluids 50(4):949-959

Pope S (2000) Turbulent Flows. Cambridge Univ. Press

Reveillon J, Demoulin FX (2007) Effects of the preferential segregation of droplets on evaporation and turbulent mixing. J Fluid Mech 583:273-302 
Seifi M, Fournier C, Grosjean N, Méès L, Marié JL, Denis L (2013) Accurate 3D tracking and size measurement of evaporating droplets using in-line digital holography and "inverse problems" reconstruction approach. Opt Express 21(23), DOI:10.1364/OE.21.027964

Sirignano WA (2010) Fluid Dynamics and Transport of Droplets and Sprays. Cambridge University Press

Soulez F, Denis L, Fournier C, Thiébaut E, Goepfert C (2007a) Inverse-problem approach for particle digital holography: accurate location based on local optimization. J Opt Soc Am A 24(4):1164-1171

Soulez F, Denis L, Thiébaut E, Fournier C, Goepfert C (2007b) Inverse problem approach in particle digital holography: out-of-field particle detection made possible. J Opt Soc Am A 24(12):3708-3716

Toschi F, Bodenschatz E (2009) Lagrangian properties of particles in turbulence. Annu Rev Fluid Mech 41:375-404 